\title{
Mandibular Osteonecrosis in a Patient Receiving Denosumab and Sunitinib
}

\author{
Ray Tanaka ${ }^{\mathrm{a}, \mathrm{e}}$, Masanao Saito ${ }^{\mathrm{b}}$, Tatsuya Abe ${ }^{\mathrm{c}}$, Hisao Ajima ${ }^{\mathrm{d}}$, Takashi Saku ${ }^{\mathrm{c}}$, \\ Takafumi Hayashi ${ }^{\mathrm{a}}$
}

\begin{abstract}
We report a case of osteonecrosis of the jaw (ONJ) in a patient undergoing concurrent treatment with the molecularly targeted agents denosumab and sunitinib for metastatic renal cancer. A 68-year-old man with multiple lung and bone metastases from renal cell carcinoma revisited our institute with exposed bone in the lingual posterior area of the right mandible. The clinical findings were consistent with ONJ according to the diagnostic criteria for bisphosphonate (BP)related ONJ, although he had not been treated with BPs. His clinical symptoms gradually worsened during conservative treatment with antibiotics; thus, he ultimately underwent surgical treatment after imaging studies confirmed osteomyelitis with necrotic bone and cellulitis of the right mandible. A definitive diagnosis of chronic osteomyelitis with sequestrum formation and no malignancy was made on the basis of histopathological findings. Follow-up clinical and radiological examinations show satisfactory postoperative recovery, and there has been no recurrence of osteonecrosis or osteomyelitis since sequestrectomy, a period of almost 2 years.
\end{abstract}

Keywords: Osteonecrosis of the jaw; Osteomyelitis; Metastatic renal cell carcinoma; Denosumab; Sunitinib; Antiresorptive agents; Antiangiogenic agents

Manuscript accepted for publication July 16, 2015

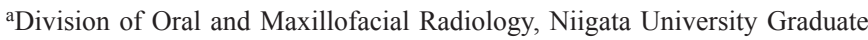
School of Medical and Dental Sciences, 2-5274 Gakkocho-dori, Chuo-ward, Niigata 951-8514, Japan

bSaito Masanao Dental Clinic, 3-33-18 Yamafutatsu, Chuo-ward, Niigata 9500922, Japan

'Division of Oral Pathology, Niigata University Graduate School of Medical and Dental Sciences, 2-5274 Gakkocho-dori, Chuo-ward, Niigata 951-8514, Japan

dAJIMA Dental Clinic, 4-2-4 Meike-kamiyama, Chuo-ward, Niigata 9500945, Japan

${ }^{\mathrm{e}}$ Corresponding Author: Ray Tanaka, Division of Oral and Maxillofacial Radiology, Niigata University Graduate School of Medical and Dental Sciences, 2-5274 Gakkocho-dori, Chuo-ward, Niigata 951-8514, Japan.

Email: renahky@dent.niigata-u.ac.jp

doi: http://dx.doi.org/10.14740/jmc2249w

\section{Introduction}

Bone metastases frequently arise from cancers of the lung, breast, prostate, and kidney. Bone metastases can complicate activities of daily living, and it is thus crucial to use bisphosphonates (BPs) or denosumab to control skeletal-related events (SRE) in patients with such metastases [1,2]. BPs have been widely used to decrease pain, the risk of pathological fracture, and the development of bone metastatic tumors. However, BPrelated osteonecrosis of the jaw (BRONJ) [3-8] is a serious complication that decreases patient's quality of life. Numerous reports of BRONJ have been published during the past decade. More recently, denosumab, which has a different mechanism of action from BPs, has been used to control SRE [1,2]; however, reports indicate that denosumab is also associated with osteonecrosis of the jaw (ONJ) [9-15]. BPs and denosumab are classified as antiresorptives, which have been associated with ONJ. In addition, some studies indicate that angiogenesis inhibitors such as bevacizumab and sunitinib might affect onset or exacerbation of ONJ [16-20]. Furthermore, recent studies indicate that concomitant use of angiogenesis inhibitors and antiresorptive agents affects ONJ incidence, exacerbation, and/or treatment outcomes [21-30].

We report a case of ONJ in a patient undergoing nearly concurrent treatment with the molecularly targeted agents denosumab and sunitinib for metastatic renal cancer.

\section{Case Report}

A 68-year-old man revisited the oral and maxillofacial surgery department of our hospital complaining of gingival irregularity in the lingual posterior region of his right mandible and tongue pain, which had persisted for about 3 weeks. His medical history included hypertension, type 2 diabetes, hypothyroidism, and multiple metastases to the right lung, left adrenal gland, left ilium, left ischium, and left femur from de novo renal cell carcinoma of the left kidney. Multiple metastatic tumors in the right lung were partially resected in 2006 . New metastases in the right lung and left suprarenal gland were discovered in 2007 , and chemotherapy with interferon was administered. Several years later the patient underwent radiotherapy for metastatic lesions in the left ilium, ischium, and femur, which were revealed by computed tomography (CT) in 2012 (Fig. 


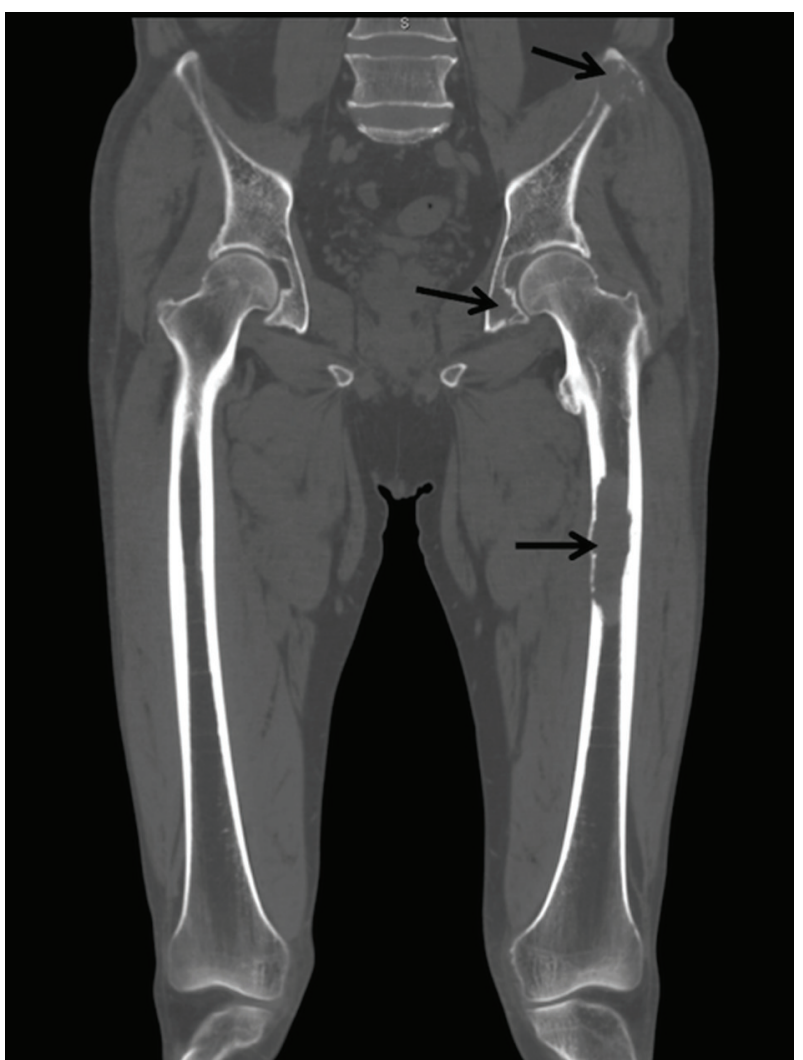

Figure 1. Metastatic lesions (arrows) in the left ilium, ischium, and femur.

1). Resection of a metastatic tumor and intramedullary nail fixation for a lesion in the left femur were performed after radiotherapy. The patient then started treatment with sunitinib (for metastatic renal cancer) and denosumab (to control SRE caused by bone lesions).

About 6 months before the present consultation, he visited our department for control of dental infection before undergoing anticancer therapy with sunitinib and denosumab. After a

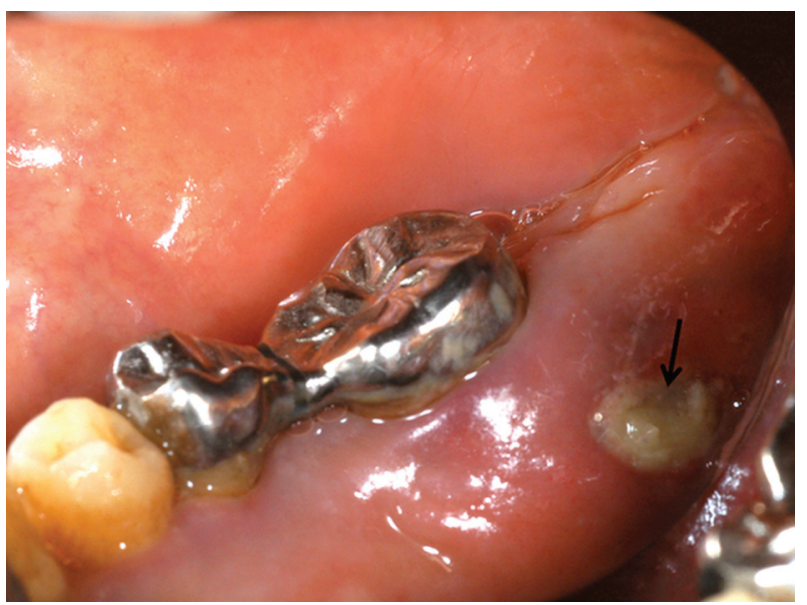

Figure 2. Intraoral photograph showing exposed bone (arrow) in the lingual posterior area of the right mandible.

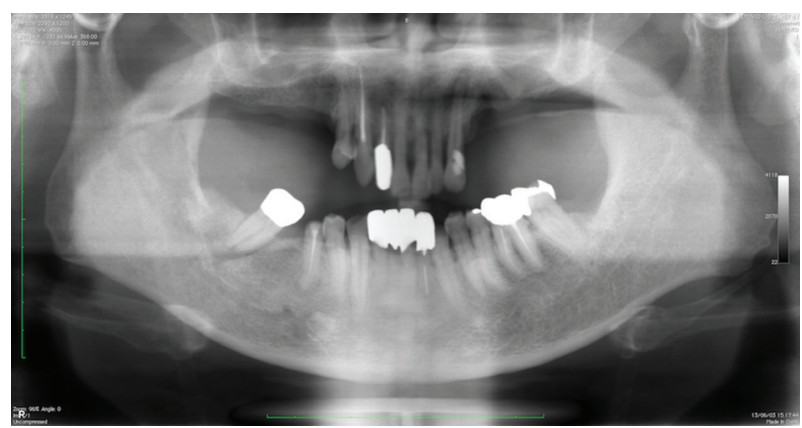

Figure 3. Panoramic radiograph at revisit showing a slightly widened periodontal ligament space in the tooth cervix, periodontal alveolar bone resorption in the area surrounding the right lower second molar, and sclerosis of cancellous bone in the molar region of the right mandible.

diagnosis of periodontitis, the right maxillar second premolar and first and second molars and left mandibular third molar were extracted in July 2012. Progress after tooth extraction was satisfactory; thus, the dental consultation was terminated and anticancer therapy was started. Sunitinib (12.5 - 50.0 mg daily) was given orally from October 2012 to March 2013. He also received once-monthly subcutaneous injections of denosumab from December 2012 through February 2013 until the development of gingival irregularity and tongue pain.

Intraoral examination at his revisit in February 2013 revealed a $10 \times 4 \mathrm{~mm}$ area of exposed, sharp-edged bone in the lingual posterior area of the right mandible (Fig. 2). The sharp edge of the necrotic bone was easily ground, without need for anesthesia. We also observed an abrasion on the right lateral margin of the tongue, which was likely due to the sharp edge of the exposed bone. A panoramic radiograph obtained on the same day revealed typical findings of periodontitis of the right mandibular second molar and sclerosis of cancellous bone in the right molar region (Fig. 3).

The exposed necrotic bone and neighboring teeth were conservatively treated for 8 weeks after the present consultation; however, surgical treatment became necessary because of

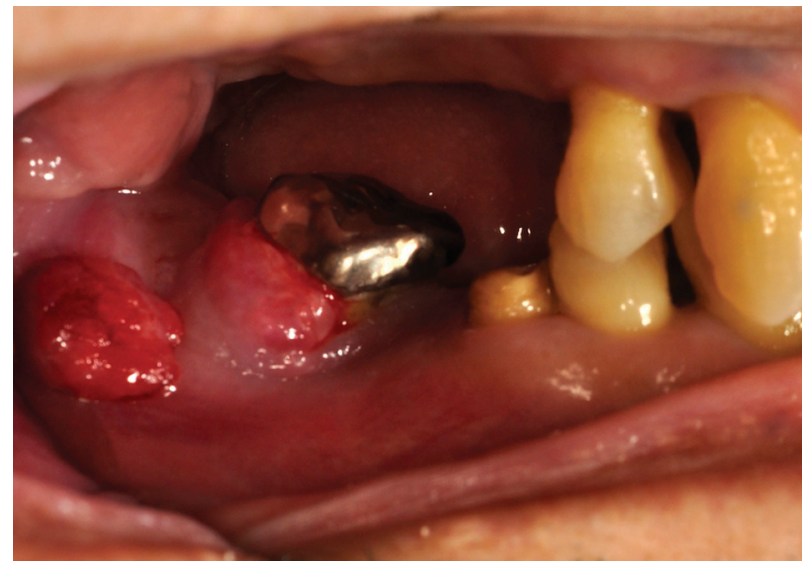

Figure 4. Intraoral photograph showing a fistula, with gingival redness and swelling, on the buccal gingiva of the mandibular right second molar. 

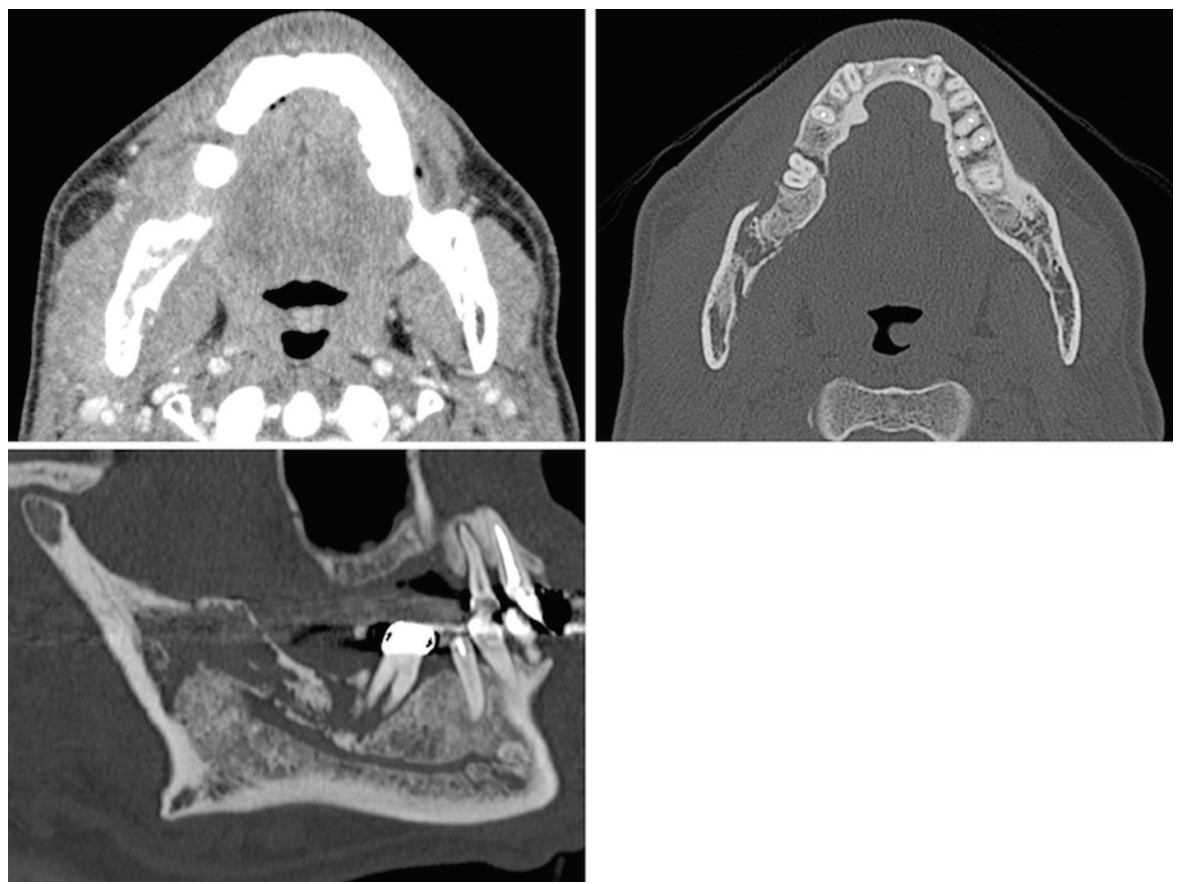

Figure 5. CT images immediately before sequestrectomy reveal characteristic findings of chronic mandibular osteomyelitis.

the gradual worsening of his clinical symptoms. The patient reported severe pain in the gingiva and right mandible, swelling in the right buccal region, and severe trismus. A red, swollen fistula was obvious in the buccal gingiva near the right mandibular second molar (Fig. 4). After several imaging studies, he underwent hyperbaric oxygen therapy before and after tooth extraction and sequestrectomy of the mandible. Radiographic images before sequestrectomy revealed sequential widening of a periodontal ligament space on the right mandibular second molar and extension of the affected region within and at the periphery of the jaw bones. A CT examination immediately before surgery revealed findings typical of chronic osteomyelitis with sequestration (Fig. 5). Magnetic resonance images indicated bone marrow edema, sequestration, inflammation of soft tissues around the right mandible, and absence of bone metastases in the mandible (Fig. 6a, b).

Histopathological analysis of specimens obtained from sequestrectomy indicated that the lesion was chronic osteomyelitis with sequestrum formation (Fig. 7). In addition, atypical histopathological features consistent with vascular endothelial cell injury were noted in specimens from the granulation tissue of affected gingiva, in contrast to the regular features of pyogenic granuloma. There was no evidence of malignancy in any specimen.

Follow-up clinical and radiological observation shows satisfactory postoperative progress, and the patient has had no recurrence of ONJ or osteomyelitis since sequestrectomy, a period of almost 2 years.

\section{Discussion}

$\mathrm{CT}$ and magnetic resonance imaging findings from our patient were consistent with chronic osteomyelitis and cellulitis of the
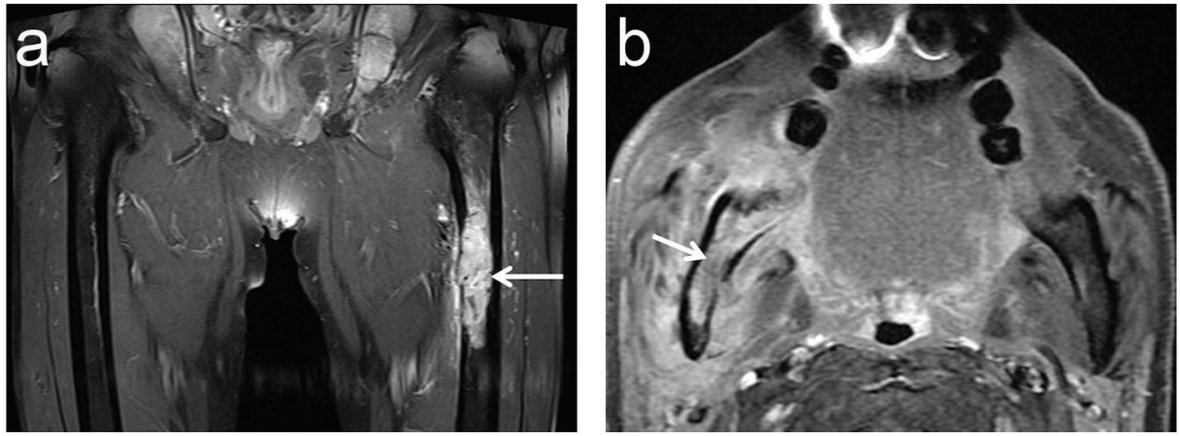

Figure 6. MR images of the right mandibular lesion and bone metastasis in the left femur. (a) Metastatic tumor in the left femur (arrow). (b) Pathological change in the bone marrow of the right mandible (arrow). 


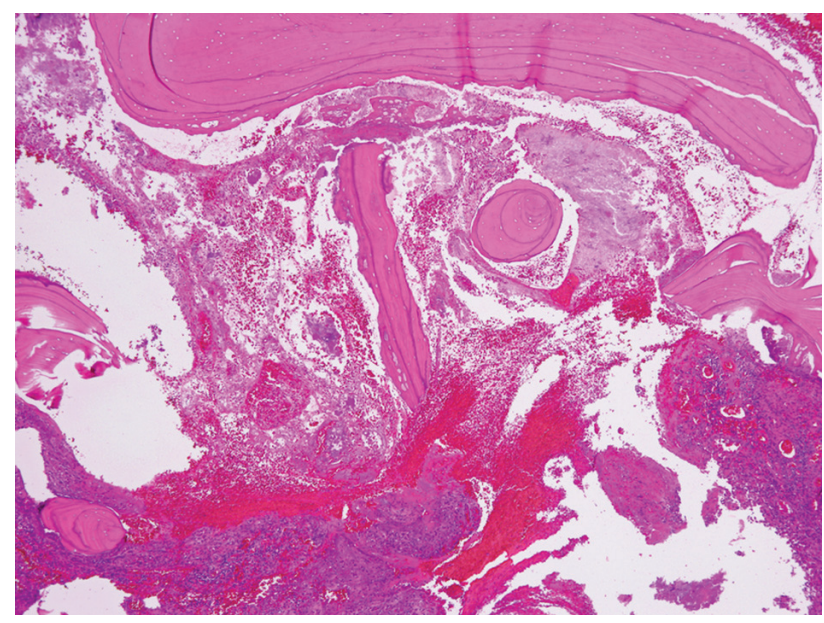

Figure 7. Histopathological specimen obtained during sequestrectomy (hematoxylin and eosin, $\times 4$ ).

mandible. We suspected that periodontal infection of the right mandibular second molar directly caused the pathological changes. Definitive histopathological diagnosis confirmed the imaging findings. Although our ultimate diagnosis was ONJ, it is difficult to determine if the present pathological condition should be classified as osteonecrosis or osteomyelitis. Because the clinical findings of our patient conformed to the diagnostic criteria of BRONJ [4, 6-8], we may have been too quick to conclude that the pathology was ONJ, even though he had never been treated with BPs. This case demonstrates the possibility of sequential aggravation of inflammatory conditions under conservative treatment and shows that hyperbaric oxygen therapy and surgical removal of inflammatory tissue, including necrotic bone, can lead to successful recovery of bone and soft tissue. These findings suggest that the pathological condition of our patient was curable mandibular osteomyelitis. A few studies have assessed treatments for patients with BRONJ and recommended surgical resection and/or hyperbaric oxygen therapy as part of multimodal therapy for patients with severe disease complicated by deep-seated soft tissue infection or refractory osteomyelitis [31-34]. Evidence regarding effective imaging modalities and treatments for ONJ has been accumulating [34]. Nevertheless, additional studies are necessary in order to clarify the pathogenesis of this disease.

Our patient was receiving denosumab, which was administered during almost the same period as sunitinib. Because our patient's initial symptoms began after the start of denosumab therapy, we assume that his condition was related to denosumab. Denosumab is a human monoclonal antibody and has specific and high affinity for human receptor activator for nuclear factor $\mathrm{\kappa B}$ ligand (RANKL). The combination of denosumab and RANKL inhibits activation of RANK and controls the formation, function, and survival of osteoclasts. Numerous recent studies have reported an association between ONJ and denosumab. Sunitinib is a peroral multitargeted tyrosine kinase inhibitor that selectively inhibits signaling pathways in various receptor tyrosine kinases which control cell proliferation, angiogenesis, and tumor metastasis. Sunitinib may inhibit the function of receptors in the innate immune system and those responsible for osteoclast differentiation. Potential causes of ONJ include not only antiresorptive agents such as BPs or denosumab but also anti-angiogenic agents such as bevacizumab, sunitinib, and sorafenib, and some researchers now use the terms medication-related osteonecrosis of the jaw (MRONJ) $[32,35]$ and drug-related osteoclastic disease of the jaw [5]. Moreover, recent studies found that concomitant use of angiogenesis inhibitors and BPs affects ONJ incidence, exacerbation, and/or treatment outcomes [21-30]. Several of these studies concluded that the combination of BPs and antiangiogenic agents may be associated with increased ONJ incidence and/or exacerbation. Although there appear to be no reports on osteonecrosis or osteomyelitis related to concurrent use of denosumab and sunitinib in the English literature, we believe that concurrent use of these drugs was the likely causes of ONJ onset and exacerbation in our patient. In addition, our patient had a long medical history, including various interventions for primary and metastatic cancer; therefore, increased susceptibility to infection may have resulted in jaw osteonecrosis or osteomyelitis. The likely pathological condition of our patient was multiple-drug-related mandibular chronic osteomyelitis. The possibility of vascular endothelial cell injury of the affected gingiva was carefully considered in histopathological analysis, as this may have been a factor in the prolongation and exacerbation of his condition. In any event, dental disease is a known risk factor for ONJ [33, 34], which strongly suggests that infection and inflammation have roles in ONJ pathogenesis. Dental infection due to periodontitis of a mandibular molar almost certainly had a substantial impact on ONJ onset and development in our patient. Obvious inflammatory findings were not observed in regions where teeth had been prophylactically extracted before administration of denosumab and sunitinib. Although we cannot identify the principal contributory factor in ONJ development, it is essential to prevent and control dental infection in patients who are compromised because of therapeutic interventions, including administration of molecular target drugs. Therefore, it is essential to establish an oral health management system based on multidisciplinary medicine or a medical network.

\section{Acknowledgement}

The authors are grateful to David Kipler, ELS for reviewing the language of the manuscript.

\section{Conflict of Interest}

The authors declare that there are no conflicts of interest.

\section{References}

1. Iranikhah M, Wilborn TW, Wensel TM, Ferrell JB. Denosumab for the prevention of skeletal-related events in patients with bone metastasis from solid tumor. Pharmacotherapy. 2012;32(3):274-284. 
2. Anghel R, Bachmann A, Beksac M, Brodowicz T, Finek J, Komadina R, Krzemieniecki K, et al. Expert opinion 2011 on the use of new anti-resorptive agents in the prevention of skeletal-related events in metastatic bone disease. Wien Klin Wochenschr. 2013;125(15-16):439-447.

3. Urade M, Tanaka N, Furusawa K, Shimada J, Shibata T, Kirita T, Yamamoto T, et al. Nationwide survey for bisphosphonate-related osteonecrosis of the jaws in Japan. J Oral Maxillofac Surg. 2011;69(11):e364-371.

4. Patel S, Choyee S, Uyanne J, Nguyen AL, Lee P, Sedghizadeh PP, Kumar SK, et al. Non-exposed bisphosphonaterelated osteonecrosis of the jaw: a critical assessment of current definition, staging, and treatment guidelines. Oral Dis. 2012;18(7):625-632.

5. Ikebe T. Pathophysiology of BRONJ: Drug-related osteoclastic disease of the jaw. Oral Science International. 2013;10:1-8.

6. Ruggiero SL, Dodson TB, Assael LA, Landesberg R, Marx RE, Mehrotra B. American Association of Oral and Maxillofacial Surgeons position paper on bisphosphonate-related osteonecrosis of the jaws--2009 update. J Oral Maxillofac Surg. 2009;67(5 Suppl):2-12.

7. Bagan JV, Hens-Aumente E, Leopoldo-Rodado M, Poveda-Roda R, Bagan L. Bisphosphonate-related osteonecrosis of the jaws: study of the staging system in a series of clinical cases. Oral Oncol. 2012;48(8):753-757.

8. Yamashita J, McCauley LK. Antiresorptives and osteonecrosis of the jaw. J Evid Based Dent Pract. 2012;12(3 Suppl):233-247.

9. Pichardo SE, Kuypers SC, van Merkesteyn JP. Denosumab osteonecrosis of the mandible: a new entity? A case report. J Craniomaxillofac Surg. 2013;41(4):e65-69.

10. Diz P, Lopez-Cedrun JL, Arenaz J, Scully C. Denosumab-related osteonecrosis of the jaw. J Am Dent Assoc. 2012;143(9):981-984.

11. Malan J, Ettinger K, Naumann E, Beirne OR. The relationship of denosumab pharmacology and osteonecrosis of the jaws. Oral Surg Oral Med Oral Pathol Oral Radiol. 2012;114(6):671-676.

12. Aghaloo TL, Dry SM, Mallya S, Tetradis S. Stage 0 osteonecrosis of the jaw in a patient on denosumab. J Oral Maxillofac Surg. 2014;72(4):702-716.

13. O'Halloran M, Boyd NM, Smith A. Denosumab and osteonecrosis of the jaws - the pharmacology, pathogenesis and a report of two cases. Aust Dent J. 2014;59(4):516519.

14. Qi WX, Tang LN, He AN, Yao Y, Shen Z. Risk of osteonecrosis of the jaw in cancer patients receiving denosumab: a meta-analysis of seven randomized controlled trials. Int J Clin Oncol. 2014;19(2):403-410.

15. Saad F, Brown JE, Van Poznak C, Ibrahim T, Stemmer SM, Stopeck AT, Diel IJ, et al. Incidence, risk factors, and outcomes of osteonecrosis of the jaw: integrated analysis from three blinded active-controlled phase III trials in cancer patients with bone metastases. Ann Oncol. 2012;23(5):1341-1347.

16. Van Poznak C. Osteonecrosis of the jaw and bevacizumab therapy. Breast Cancer Res Treat. 2010;122(1):189-191.

17. Fleissig Y, Regev E, Lehman H. Sunitinib related oste- onecrosis of jaw: a case report. Oral Surg Oral Med Oral Pathol Oral Radiol. 2012;113(3):e1-3.

18. Koch FP, Walter C, Hansen T, Jager E, Wagner W. Osteonecrosis of the jaw related to sunitinib. Oral Maxillofac Surg. 2011;15(1):63-66.

19. Nicolatou-Galitis O, Migkou M, Psyrri A, Bamias A, Pectasides D, Economopoulos T, Raber-Durlacher JE, et al. Gingival bleeding and jaw bone necrosis in patients with metastatic renal cell carcinoma receiving sunitinib: report of 2 cases with clinical implications. Oral Surg Oral Med Oral Pathol Oral Radiol. 2012;113(2):234-238.

20. Troeltzsch M, Woodlock T, Kriegelstein S, Steiner T, Messlinger K. Physiology and pharmacology of nonbisphosphonate drugs implicated in osteonecrosis of the jaw. J Can Dent Assoc. 2012;78:c85.

21. Brunello A, Saia G, Bedogni A, Scaglione D, Basso U. Worsening of osteonecrosis of the jaw during treatment with sunitinib in a patient with metastatic renal cell carcinoma. Bone. 2009;44(1):173-175.

22. Hoefert S, Eufinger H. Sunitinib may raise the risk of bisphosphonate-related osteonecrosis of the jaw: presentation of three cases. Oral Surg Oral Med Oral Pathol Oral Radiol Endod. 2010;110(4):463-469.

23. Guarneri V, Miles D, Robert N, Dieras V, Glaspy J, Smith I, Thomssen C, et al. Bevacizumab and osteonecrosis of the jaw: incidence and association with bisphosphonate therapy in three large prospective trials in advanced breast cancer. Breast Cancer Res Treat. 2010;122(1):181-188.

24. Christodoulou C, Pervena A, Klouvas G, Galani E, Falagas ME, Tsakalos G, Visvikis A, et al. Combination of bisphosphonates and antiangiogenic factors induces osteonecrosis of the jaw more frequently than bisphosphonates alone. Oncology. 2009;76(3):209-211.

25. Smidt-Hansen T, Folkmar TB, Fode K, Agerbaek M, Donskov F. Combination of zoledronic Acid and targeted therapy is active but may induce osteonecrosis of the jaw in patients with metastatic renal cell carcinoma. J Oral Maxillofac Surg. 2013;71(9):1532-1540.

26. Beuselinck B, Wolter P, Karadimou A, Elaidi R, Dumez H, Rogiers A, Van Cann T, et al. Concomitant oral tyrosine kinase inhibitors and bisphosphonates in advanced renal cell carcinoma with bone metastases. Br J Cancer. 2012;107(10):1665-1671.

27. Fusco V, Porta C, Saia G, Paglino C, Bettini G, Scoletta M, Bonacina R, et al. Osteonecrosis of the Jaw in Patients With Metastatic Renal Cell Cancer Treated With Bisphosphonates and Targeted Agents: Results of an Italian Multicenter Study and Review of the Literature. Clin Genitourin Cancer. 2015;13(4):287-294.

28. Lescaille G, Coudert AE, Baaroun V, Ostertag A, Charpentier E, Javelot MJ, Toledo R, et al. Clinical study evaluating the effect of bevacizumab on the severity of zoledronic acid-related osteonecrosis of the jaw in cancer patients. Bone. 2014;58:103-107.

29. Ayllon J, Launay-Vacher V, Medioni J, Cros C, Spano JP, Oudard S. Osteonecrosis of the jaw under bisphosphonate and antiangiogenic therapies: cumulative toxicity profile? Ann Oncol. 2009;20(3):600-601.

30. Ngamphaiboon N, Frustino JL, Kossoff EB, Sullivan 
MA, O'Connor TL. Osteonecrosis of the jaw: dental outcomes in metastatic breast cancer patients treated with bisphosphonates with/without bevacizumab. Clin Breast Cancer. 2011;11(4):252-257.

31. Bedogni A, Saia G, Bettini G, Tronchet A, Totola A, Bedogni G, Ferronato G, et al. Long-term outcomes of surgical resection of the jaws in cancer patients with bisphosphonate-related osteonecrosis. Oral Oncol. 2011;47(5):420-424.

32. Ristow O, Otto S, Troeltzsch M, Hohlweg-Majert B, Pautke C. Treatment perspectives for medication-related osteonecrosis of the jaw (MRONJ). J Craniomaxillofac Surg. 2015;43(2):290-293.

33. Freiberger JJ, Padilla-Burgos R, McGraw T, Suliman HB, Kraft KH, Stolp BW, Moon RE, et al. What is the role of hyperbaric oxygen in the management of bisphosphonate-related osteonecrosis of the jaw: a randomized controlled trial of hyperbaric oxygen as an adjunct to surgery and antibiotics. J Oral Maxillofac Surg. 2012;70(7):15731583.

34. Khan AA, Morrison A, Hanley DA, Felsenberg D, McCauley LK, O'Ryan F, Reid IR, et al. Diagnosis and management of osteonecrosis of the jaw: a systematic review and international consensus. J Bone Miner Res. 2015;30(1):3-23.

35. Ruggiero SL, Dodson TB, Fantasia J, Goodday R, Aghaloo T, Mehrotra B, O'Ryan F. American Association of Oral and Maxillofacial Surgeons position paper on medication-related osteonecrosis of the jaw--2014 update. J Oral Maxillofac Surg. 2014;72(10):1938-1956. 